\title{
Australasian Journal OF LogiC
}

\section{Relevant ARIthmetic}

\author{
Robert K. Meyer
}

Australian National University

Let $L$ be a relevant logic; e.g., the system RQ of [5]. One forms the corresponding first-order Peano arithmetic $L^{\sharp}$, with $0, \times,+,{ }^{\prime}$ (successor) as non-logical constants, by adding the usual arithmetical axioms. E.g., form $R^{\sharp}$ from $R Q$ by taking the following as non-logical axioms:

$$
\begin{array}{ll}
\# 1 & x=y \leftrightarrow x^{\prime}=y^{\prime} \\
\# 2 & x=y \rightarrow \cdot x=z \rightarrow y=z \\
\# 3 & x^{\prime} \neq 0 \\
\# 4 & x+0=x \\
\# 5 & x+y^{\prime}=(x+y)^{\prime} \\
\# 6 & x \times 0=0 \\
\# 7 & x \times y^{\prime}=(x \times y)+x
\end{array}
$$

Not all forms of the mathematical induction principle are relevantly valid. A sufficient form of this principle, however, is the rule $R M I$ : "From $A 0$ and $A x \rightarrow A x^{\prime}$, infer $A x . "$

$\mathrm{R}^{\sharp}$, as formulated with \#1-\#7, has the interesting property of being trivially absolutely consistent. In fact, consider the model of $\mathrm{R}^{\sharp}$, in the 3-point Sugihara matrix of [1], p. 403, when the domain of the model is taken as the integers modulo 2. On the natural assignment of arithmetical values in the integers modulo 2 to the non-logical constants, then setting the Sugihara value of $0=0$ and $0^{\prime}=0^{\prime}$ as 0 and of $0=0^{\prime}$ and $0^{\prime}=0$ as -1 , determining values on complex formulas in the natural Sugihara way, it is readily observed that all theorems of $\mathrm{R}^{\sharp}$ take one of the values $0,+1$ in the model, 
while $0=0^{\prime}$ takes the value -1 . This is evidently a finitary proof refuting $0=1$ in first-order arithmetic, as Hilbert desired. (Moral: he should have used $R^{\sharp}$.) Similarly, every other incorrect quantifier-free equation (which may contain variables) may be refuted. In this sense, accordingly, $R^{\sharp}$ (like certain cut-free formulations of classical arithmetic, but with the proviso that $\mathrm{R}^{\sharp}$ is formulated naturally with modus ponens for $\rightarrow$ ) avoids Gödel's second theorem. I.e., one can prove, in the natural way, within $R^{\sharp}$ that it is arithmetically consistent, in the sense that equations which violate the addition or multiplication tables for + and $\times$ are trivially undemonstrable. But the impossibility of proving within $\mathrm{R}^{\sharp}$ that $\mathrm{R}^{\sharp}$ is negation-consistent is as in the classical case.

Both intuitionist and classical Peano arithmetic (e.g., as in [2]) may be developed within $R^{\sharp}$ on well-motivated translations. Accordingly, classical metatheory - as it involves, e.g., recursive functions and relations - may be developed within $R^{\sharp}$. It follows, e.g., that $R^{\sharp}$ is essentially undecidable in a Tarskian sense; i.e., all its negation-consistent extensions are undecidable (provided, of course, that it is consistent). But the structure of $\mathrm{R}^{\sharp}$-theories is much richer than in the classical case; inconsistent but non-trivial theories in particular are admitted, and some of these may be decidable. (Essentially, one such was just exhibited in setting out the basic arithmetical consistency argument.)

Although, in a sense, $R^{\sharp}$ contains intuitionist Peano arithmetic (and in particular licenses, on translation, all intuitionist arguments, though not, in the direct sense, all intuitionist theorems), it is much more closely related to the classical system. Classical arithmetic $P^{\sharp}$ is contained in $R^{\sharp}$ on exact and homomorphic translation, but not necessarily on the direct translation. Question: are there formulas of the vocabulary of $P^{\sharp}$ (i.e., not containing any relevant implications) which are theorems of $P^{\sharp}$ but not $R^{\sharp}$ ? Answer: I don’t know. For large classes of formulas of $\mathrm{P}^{\sharp}$, it is possible to show that they will be theorems of $\mathrm{P}^{\sharp}$ iff they are theorems of $\mathrm{R}^{\sharp}$; these include, e.g., the elementary arithmetical theorems proved for $\mathrm{P}^{\sharp}$ formally in [2]. Caution: some of these questions are delicate; relevant counterparts of some classical laws - e.g., involving cancellation - fail, for what seem to be good relevant reasons (though this view is subject to change), when they are inaccurately formulated. But, in the classical vocabulary, all those investigated that hold classically hold relevantly.

It is to be noted that it would suffice (though it is not necessary) to show that $P^{\sharp}$ is exactly contained in $R^{\sharp}$ on the natural translation to show that

Australasian Journal of Logic (18:5) 2021, Article no. 3 
Ackermann's rule $\gamma$ (modus ponens for material implication) is admissible in $R^{\sharp}$. This is an old problem for relevant theories (cf., [1]) which arises anew on each extension of insight. Note that the admissibility of $\gamma$ for $R^{\sharp}$, in view

of its arithmetical consistency, implies the negation-consistency not only of $R^{\sharp}$ but of $P^{\sharp}$. Accordingly, for reasons like those raised in [3], a proof of $\gamma$ for $R^{\sharp}$, if there is one, cannot be elementary. Since $R^{\sharp}$ itself is incomplete, for Gödelian reasons, it may not have the necessary stability to admit $\gamma$. But I have no conjecture either way. (I do note, however, that if $\mathrm{R}^{\sharp}$ is enriched with an $\omega$-rule (from $A n$ for each numeral $n$, infer $\forall x A x$ ), to form a system $\mathrm{R}_{\omega}$, then it will admit $\gamma$ for all formulas (including statements involving relevant $\rightarrow$, on which even $R_{\omega}$ remains incomplete, while including all the intuitive arithmetic truths in its classical part).

Research has just begun into application of the relevant logics developed in [1] and elsewhere. However it seems clear, even in the initial stages, that both new insights and new technical results, even for classical systems, are to be expected from this research. Note that one is not wedded to $\mathrm{R}$ for the development of these theories; the systems E and RM of [1] seem equally appropriate, and perhaps in some ways more appropriate. And I am particularly indebted, in setting out both technical material and comments thereon, to J. Michael Dunn, with whom I have discussed the issues arising out of the application of relevant logics - to arithmetic in particular - over a number of years, and whose ideas have played a continuing role in developments here. A detailed development of the present material will be found in [4], and in some successor volume to [1].

\section{Editors' Acknowledgments}

This abstract was previously published as:

- R. K. Meyer. Relevant arithmetic. Bulletin of the Section of Logic, 5:133-137, 1976.

and is included in this volume with the permission of the Bulletin of the Section of Logic, and Łodz University Press.

Australasian Journal of Logic (18:5) 2021, Article no. 3 


\section{References}

[1] A. R. Anderson and N. D. Belnap. Entailment, volume I. Princeton University Press, Princeton, 1975.

[2] S. C. Kleene. Introduction to Metamathematics. North-Holland, New York, 1952.

[3] G. Kreisel. A survey of proof theory. Journal of Symbolic Logic, 33(3):321-388, 1968. Esp. p. 332, footnote.

[4] R. K. Meyer. The consistency of arithmetic: A relevant formulation. In preparation, 1976.

[5] R. K. Meyer and H. Leblanc. A semantical completeness for relevant quantification theories. Journal of Symbolic Logic, 35(1):353, 1970. But the system RQ is due to the authors of [1].

Australasian Journal of Logic (18:5) 2021, Article no. 3 